\title{
Eosinophilic Meningitis and Intracranial Haemorrhage: Can it be Neuro-Gnathostomiasis?
}

\author{
Basnet S', Shrestha $L^{2}$, Paudel DR
}

\begin{abstract}
Peripheral eosinophilia is commonly encountered in our clinical practice. In this case report, we present a child who presented with hemorrhagic stroke and was finally diagnosed of eosinophilic meningitis. In this case report, we also propose probable etiologic agent.
\end{abstract}

\section{Introduction}

Eosin osinophilic meningitis (EM) is an uncommon diagnosis in a child - presenting with cerebral parenchymal haemorrhage. EM is defined as the presence of 10 eosinophils $/ \mathrm{mL}$ in CSF or at least $10 \%$ eosinophils in the total CSF leukocyte count based on the studies done in the past ${ }^{1}$.Physician's unawareness of clinical symptoms, absence of signs of meningeal irritation and failure of laboratory tests to identify eosinophilia in CSF makes the diagnosis of eosinophilic meningitis challenging ${ }^{2}$. The presence of eosinophils in CSF suggests number of infectious and non-infectious etiologies ${ }^{3}$. In a previously healthy child, the commonest causes of eosinophilic meningitis are neuroparasitesangiostrongyliasis (rat lung worm) and gnathostomiasis worldwide ${ }^{4}$

\section{The Case}

Twelve years old boy from Dolakha, Nepal presented with sudden onset of weakness of left upper and lower limb for one day which was preceded by severe headache. There was no vomiting, loss of consciousness, any abnormal body movements or history suggestive of cranial nerve involvement. He was developmentally normal child without any significant illnesses in the past. On arrival, his vital signs were normal. His higher mental function and cranial nerve examination was normal. He had decreased tone in left upper and lower limbs with power of 2/5 on all groups of muscles of left upper limb and 1/5 on all group of muscles on the left lower limb. Reflexes were brisk with extensor plantar on left side. Motor examination of right side was normal. His sensory examination, cerebellar examinations were normal. There was no sign of meningeal irritation and other systemic examination were normal.

His CT scan revealed haemorrhage in right frontal region (Figure 1). His haemoglobin was $12 \mathrm{gm} \%$. PT:14, INR:1.16 (Control:12), APTT: 30 (Control: 30) and CT angiography was normal. The power of both limbs gradually improved over next few days but suddenly on $10^{\text {th }}$ day of hospital admission, he again developed headache and
${ }^{1}$ Dr. Srijana Basnet, MBBS. MD, Assistant Professor. ${ }^{2}$ Dr. Laxman Shrestha, MBBS. MD, Professor. ${ }^{3} \mathrm{Dr}$. Daman Raj Poudel, MBBS. MD. Assistant Professor. All from the Department of Paediatrics, Tribhuvan University Teaching Hospital, Institue of Medicine, Maharajgunj, Kathmandu, Nepal.

\section{Address for correspondence \\ Dr. Srijana Basnet \\ E-mail: drsrijanabasnet@yahoo.com}

\section{How to cite}

Basnet S, Shrestha L, Paudel DR. Eosinophilic Meningitis and Intracranial Haemorrhage: Can it be Neuro-Gnathostomiasis ?. J Nepal Paediatr Soc 2018;38(2):125-127.

doi: http://dx.doi.org/10.3126/jnps.v38i2.19211

This work is licensed under a Creative Commons Attribution 3.0 License.

$$
\text { (c) (i) }
$$


multiple episodes of vomiting without signs of meningeal irritation. He was managed with mannitol and 3\% Normal Saline. His WBC: $11,330 /$ cumm with (N:50\% L:22\% and $E: 28 \%$ ) with absolute eosinophilia count (AEC): $2847 \mathrm{cu} / \mathrm{mm}$ so Cerebrospinal fluid analysis(CSF) was done which analysis revealed Total Cells: of 210 cells/ $\mathrm{mm}^{3}$ with 90\% Monomorphs and 10\% Polymorphs, Sugar: 55 mg/dl, Protein: 58 mg/dl, RBC:500 / $\mathrm{mm}^{3}$. CSF Eosinophil was $40 \%$.

On further review of history, it was also found that he ate raw fish caught at the nearby river frequently. There was also history of abdominal wall swelling a month back which resolved by itself. His stool examination revealed eggs of Trichuristrichuria and hookworm. He was treated with prednisolone followed by three week course of Albendazole, Ivermectin and Praziquantel. Physiotherapy was given and discharged home after 16 days. On follow up he completely improved.

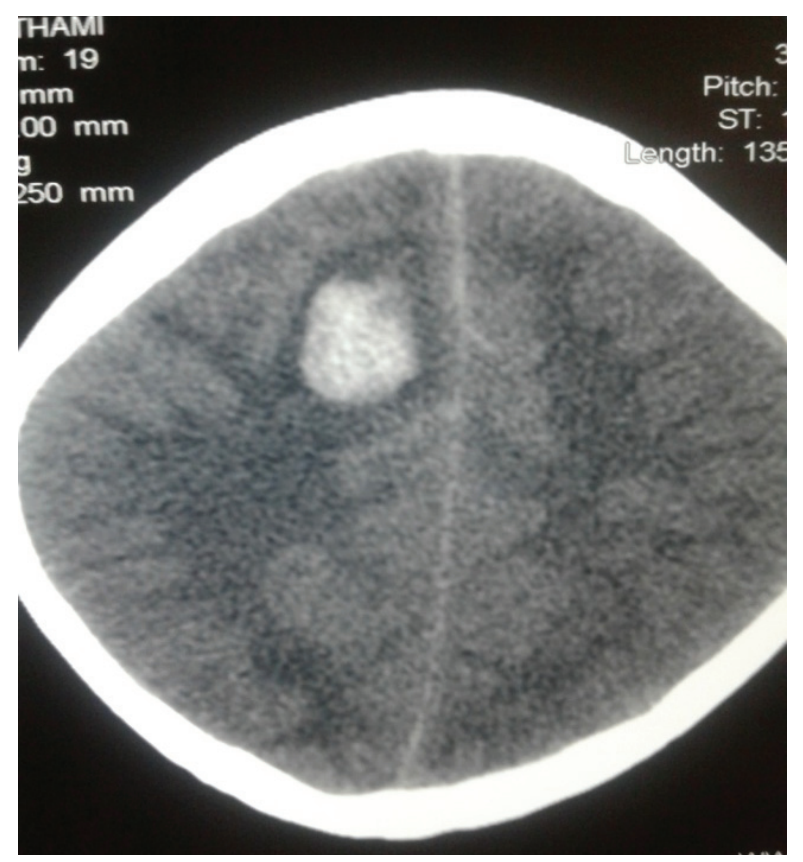

Fig 1: CT scan showing intracerebral haemorrhage in right frontal lobe

\section{Discussion}

Though there are few case series of parasitic infestation in $\mathrm{Nepal}^{5,6}$ neurological manifestation of parasitic infestation had not been described in any of these reports. Though there are few studies on peripheral eosinophilia and acute encephalitis syndrome from Nepal, none of these include or report cases of eosinophilic meningitis. Thus, to our knowledge this is a first reported case of eosinophilic meningitis from Nepal.

The recurrence of symptoms in presence of peripheral eosinophilia makes us suspect eosinophilic meningitis in our patient so CSF analysis was done in spite of absence of signs of meningeal irritation. Eosinophils can be distorted or destroyed during CSF processing and can be mistaken for neutrophils if CSF analysis is automated ${ }^{7}$ Therefore, if there is a suspicion for eosinophilic meningitis, the sample should be properly stained with Wright or Giemsa staining and should be manually read. He was a previously healthy child with no risk factor or pointer towards a non-parasitic cause of eosinophilic meningitis. (Immunodeficiency, exposure to drugs, contact with or evidence of tuberculosis) so the parasitic infestation was considered to be the cause for this EM. Angiostrongylus spp. infection is usually seen as self-limited eosinophilic meningitis and only rarely causes severe disease with prominent spinal or cerebral involvement ${ }^{8}$ which makes this diagnosis unlikely in our patient. A history of consumption of raw freshwater fish, intracranial haemorrhage $(\mathrm{ICH})$ on neuroimaging and Eosinophilic meningitis (EM) would suggest the diagnosis of neuro-gnathostomiasis if immunodiagnostic testing is not available 9.10 .

Cutaneous gnathostomiasis is the most common manifestation of infection. The self-resolving abdominal wall swelling in our patient probably was cutaneous gnathostomiasis. The clinical symptoms of neurognathostomiasis are related to mechanical disruption by larvae migration in CNS, ${ }^{11}$ relapse may occur and progression of symptomatology may not cease if larvae are not killed by anthelminthic agents ${ }^{12}$. Hence we treated our patient with anthelminthic and steroid to alleviate risk of increasing cerebral oedema due to dying neuro-parasites. Although steroids have also been used to relieve perifocal oedema, no well-controlled studies have shown proven efficacy ${ }^{2,13}$.

In the systemic review done by Devleesschauwe Brecht et $\mathrm{al}^{14}$ on parasitic zoonosis in Nepal, only Angiostrongyluscantonensis was detected. However, G. spinigeruminfection is endemic in Southeast Asia, particularly in Thailand and Taiwan ${ }^{15,16,17}$. Since, Nepal also share similar geographic location and human behaviour of eating uncooked animal food make us suspect that this parasite must be prevalent in our part too. The prevalence of this parasite in our part need to be further researched.

\section{Conclusion}

We proposed gnathostoma species for eosinophilic meningitis and intracranial haemorrhage. We also highlighted the diagnostic challenges for eosinophilic meningitis and neuro-gnathostomiasis. This case also highlighted the probable existence of neuro-parasites except neuro-cysticercosis in our part of the globe 


\section{References}

1. Irani DN. Cerebrospinal fluid in Clinical Practice. Saunders: Philadelphia; 2009. p. 278

2. Graeff-Teixeira C, da Silva AC, Yoshimura K. Update on eosinophilic meningoencephalitis and its clinical relevance. Clin Microbiol Rev 2009;22:322e48. DOI: doi: [10.1128/CMR.00044-08]

3. Lo Re V, Gluckman SJ. Eosinophilic meningitis. Am J Med 2003;114:217-23. DOI: https://doi.org/10.1016/ S0002-9343(02)01495-X

4. Ramirez-Avila L, Slome S, Schuster F, Gavali S, Schantz P, Sejvar J, et al. Eosinophilic Meningitis due to AngiostrongylusandGnathostoma Species. Clin Infect Dis 2009;48:322-7. DOI: 10.1086/595852.

5. Shrestha $S$, Timila $D$, Karki $U$ et al Clinical profile of children with moderate-to-severe eosinophilia presenting to a tertiary hospital in Nepal. Trop Doc 2012;42:232-34. DOI: 10.1258/td.2012.120284.

6. Shrestha S, Dongol Singh S, Shrestha NC, Shrestha RPB . Clinical and Laboratory Profile of Children with Eosinophilia at Dhulikhel Hospital. Kathmandu Univ Med J 2012;38(2):58-62.

7. Kuberski T. Eosinophils in cerebrospinal fluid. Ann Intern Med 1979;91:70-5. DOI: 10.7326/0003-481991-1-70

8. Nawa Y, Hatz C, Blum J. Sushi delights and parasites: the risk of fish borne and food borne parasitic zoonoses in Asia. Clin Infect Dis 2005;41:1297-303. DOI: doi:10.1086/496920

9. Punyagupta S, Bunnag T, Juttijudata P. Eosinophilic meningitis in Thailand. Clinical and epidemiological characteristics of 162 patients with myeloencephalitis probably caused by Gnathostomaspinigerum. J Neurol Sci 1990;96:241e56.
10. Ramirez-Avila L, Slome S, Schuster FL, Gavali S, Schantz PM, Sejvar J, et al. Eosinophilic meningitis due to Angiostrongylus and Gnathostoma species. Clin Infect Dis 2009;48:322e7. DOI: $10.1086 / 595852$.

11. Sithinamsuwan $P$, Chairangsaris $P$. Images in clinical medicine. Gnathostomiasisdneuroimaging of larval migration. N Engl J Med 2005;353:188. DOI: 10.1056/ NEJMicm040795

12. Schmutzhard E, Boongird P, Vejjajiva A. Eosinophilic meningitis and radiculomyelitis in Thailand, caused by CNS invasion of Gnathostomaspinigerum and Angiostrongyluscantonensis. J Neurol Neurosurg Psychiatr 1988;51:80e7.

13. Katchanov J, Sawanyawisuth K, Chotmongkoi V, Nawa Y. Neurognathostomiasis, a neglected parasitosis of the central nervous system. Emerg Infect Dis 2011;17:1174e80. DOI: [10.3201/eid1707.101433]

14. Devleesschauwer $B$, Ale A, Torgerson $P$ et al. The Burden of Parasitic Zoonoses in Nepal: A Systematic Review. PLOS Neglected Trop Dis 2014;8(1);e2634. DOI: https://doi.org/10.1371/journal.pntd.0002634

15. Rusnak JM, Lucey DR. Clinical gnathostomiasis: case report and review of the English-language literature. Clin Infect Dis1993;16:33-50.

16. Hung $M N$, Huang $H$, Dekumyoy $P$ et al. First case of neurognathostomiasis in TaiwandA Thai laborer presenting with eosinophilic meningitis and intracranial hemorrhage. J Formosan Med Assoc 2015;114:1280e1284. DOI: https://doi.org/10.1016/j. jfma.2013.07.006

17. Moore DAJ, McCroddan J, Dekumyoy P, Chiodin PL. Gnathostomiasis:an emerging imported disease. Emerg Infect Dis 2003;9:647-50. DOI: 10.3201/ eid0906.020625 